\title{
The kinematic recovery process of rhesus monkeys after spinal cord injury
}

\author{
Rui-Han $\mathrm{WEI}^{1)}$, Can $\mathrm{ZHAO}^{1,2,3)}$, Jia-Sheng RAO ${ }^{1,2,4) *}$, Wen $\mathrm{ZHAO}^{5}$, Xia ZHOU ${ }^{1)}$, Peng-Yu TIAN ${ }^{1)}$, \\ Wei SONG ${ }^{6)}$, Run JI ${ }^{7)}$, Ai-Feng ZHANG ${ }^{8)}$, Zhao-Yang YANG ${ }^{1.5) *}$, and Xiao-Guang $\mathrm{LI}^{1,2,5) *}$ \\ 1) Beijing Key Laboratory for Biomaterials and Neural Regeneration, School of Biological Science and Medical \\ Engineering, Beihang University, No. 37 Xueyuan Road, Haidian District, Beijing 100083, P.R. China \\ ${ }^{2}$ Beijing International Cooperation Bases for Science and Technology on Biomaterials and Neural Regeneration, \\ Beijing Advanced Innovation Center for Big Data-Based Precision Medicine, Beihang University, No. 37 Xueyuan \\ Road, Haidian District, Beijing 100083, P.R. China \\ ${ }^{3)}$ School of Instrument Science and Opto-Electronic Engineering, Beihang University, No. 37 Xueyuan Road, \\ Haidian District, Beijing 100083, P.R. China \\ 4) Beijing Advanced Innovation Center for Biomedical Engineering, Beihang University, No. 37 Xueyuan Road, \\ Haidian District, Beijing 100083, P.R. China \\ ${ }^{5)}$ Department of Neurobiology, Capital Medical University, No. 10 Xitoutiao Road, Youanmenwai, Xicheng District, \\ Beijing 100191, P.R. China \\ ${ }^{6)}$ Rehabilitation Engineering Research Institute, China Rehabilitation Research Center, No. 10 Jiaomenbei Road, \\ Fengtai District, Beijing 100068, P.R. China \\ 7) Human Biomechanics Laboratory, National Research Center for Rehabilitation Technical Aids, No. 1 \\ Ronghuazhong Road, Daxing District, Beijing 100176, P.R. China \\ ${ }^{8)}$ Beijing Friendship Hospital, Capital Medical University, No. 95 Yongan Road, Xicheng District, Beijing 100050, \\ P.R. China
}

\begin{abstract}
After incomplete spinal cord injury ( $\mathrm{SCl}$ ), neural circuits may be plastically reconstructed to some degree, resulting in extensive functional locomotor recovery. The present study aimed to observe the post-SCl locomotor recovery of rhesus monkey hindlimbs and compare the recovery degrees of different hindlimb parts, thus revealing the recovery process of locomotor function. Four rhesus monkeys were chosen for thoracic hemisection injury. The hindlimb locomotor performance of these animals was recorded before surgery, as well as 6 and 12 weeks post-lesion. Via principal component analysis, the relevant parameters of the limb endpoint, pelvis, hindlimb segments, and
\end{abstract}

(Received 8 February 2018 / Accepted 16 April 2018 / Published online in J-STAGE 16 May 2018)

Addresses corresponding: X.-G. Li, Beijing Key Laboratory for Biomaterials and Neural Regeneration, School of Biological Science and Medical Engineering, Beihang University, No. 37 Xueyuan Road, Haidian District, Beijing 100083, P.R. China; Beijing International Cooperation Bases for Science and Technology on Biomaterials and Neural Regeneration, Beijing Advanced Innovation Center for Big Data-Based Precision Medicine, No. 37 Xueyuan Road, Haidian District, Beijing 100083, P.R. China; Department of Neurobiology, School of Basic Medical Sciences, Capital Medical University, No. 10 Xitoutiao Road, Youanmenwai, Xicheng District, Beijing 100191, P.R.China

J.-S. Rao, Beijing Key Laboratory for Biomaterials and Neural Regeneration, School of Biological Science and Medical Engineering, Beihang University, No. 37 Xueyuan Road, Haidian District, Beijing 100083, P.R. China; Beijing International Cooperation Bases for Science and Technology on Biomaterials and Neural Regeneration, Beijing Advanced Innovation Center for Big Data-Based Precision Medicine, No. 37 Xueyuan Road, Haidian District, Beijing 100083, P.R. China; Beijing Advanced Innovation Center for Biomedical Engineering, Beihang University, No. 37 Xueyuan Road, Haidian District, Beijing 100083, P.R. China

Z.-Y. Yang, Department of Neurobiology, School of Basic Medical Sciences, Capital Medical University, No. 10 Xitoutiao Road, Youanmenwai, Xicheng District, Beijing 100191, P.R.China or Beijing Key Laboratory for Biomaterials and Neural Regeneration, School of Biological Science and Medical Engineering, Beihang University, No. 37 Xueyuan Road, Haidian District, Beijing 100083, P.R. China

*These corresponding authors contributed equally.

Supplementary Methods and Table: refer to J-STAGE: https://www.jstage.jst.go.jp/browselexpanim

(c) $(1)$ This is an open-access article distributed under the terms of the Creative Commons Attribution Non-Commercial No Derivatives
(by-nc-nd) License $<$ http://creativecommons.org/licenses/by-nc-nd/4.0/>. (C2018 Japanese Association for Laboratory Animal Science 
joints were processed and analyzed. Twelve weeks after surgery, partial kinematic recovery was observed at the limb endpoint, shank, foot, and knee joints, and the locomotor performance of the ankle joint even recovered to the pre-lesion level; the elevation angle of the thigh and hip joints showed no obvious recovery. Generally, different parts of a monkey hindlimb had different spontaneous recovery processes; specifically, the closer the part was to the distal end, the more extensive was the locomotor function recovery. Therefore, we speculate that locomotor recovery may be attributed to plastic reconstruction of the motor circuits that are mainly composed of corticospinal tract. This would help to further understand the plasticity of motor circuits after spinal cord injury.

Key words: locomotion, monkey, spinal cord injury, spontaneous recovery

\section{Introduction}

The spinal cord is part of the central nervous system and serves as a connecting pathway between the brain and peripheral nervous system. Spinal cord injury (SCI) physically destroys neural circuits at the lesion site, usually causing the loss of motor and sensory functions beneath the injury site. After incomplete SCI, neural circuits have plasticity to some degree $[5,16,17,34]$ that plays a role in facilitating the recovery of sensory and motor function [27]. Neural plasticity has been demonstrated in incomplete spinal cord injury rodents $[4,30$, $33,35]$ and cats $[18,20]$. For instance, Weidner et al. reported that after bilateral dorsal corticospinal transection of the rat cervical spinal cord, spontaneous sprouting from the ventral corticospinal tract occurred and was paralleled by functional recovery of rat forelimbs [32]. Martinez et al. [19] found that for a thoracic hemisection injury cat, treadmill training may facilitate spontaneous compensatory changes of the spinal circuitry and consequently promote locomotor recovery. Nevertheless, the post-SCI locomotor recovery of these animal models is quite different from that of human beings because they have different spinal cord structures [11]. Let us take the corticospinal tract (CST) that controls spontaneous movement in humans as an example. In rodents, the CST is mainly located in the dorsal column, originating from the contralateral motor cortex [11]. Compared with rats, the lateral CST of cats is located in the lateral funiculi, representing appreciable amounts of contralateral preand postcruciate cortex [28]. Approximately $88 \%$ of corticospinal axons in primates decussate along the spinal cord midline, representing appreciable amounts of contralateral peri-Rolandic cortex; primates are more similar to humans than cats $[11,15]$. A significant proportion of CST fibers project to the ventral horn, and some axons synapse directly on motoneurons.

With a similar spinal cord structure to humans, nonhuman primates have been commonly used to explore functional recovery after SCI [6, 23, 24, 29]. Babu et al. [3] reported that 4-7 months after thoracic hemisection injury, parameters such as toe spread and intermediary toes of the monkey footprint recovered to the pre-lesion level. Rosenzweig et al. [26] observed that six months after cervical cord hemisection, movement of monkey forelimbs and hindlimbs had recovered to some degree. The study by Friedli et al. [11] revealed the critical role of reorganization of the corticospinal tract in functional locomotor recovery in lateral SCI rats and monkeys. All of these investigations, however, were less likely to display the different recovery processes of each limb part. Actually, distal and proximal muscles do not have exactly the same motor control circuits. Distal musculature is mainly controlled by the corticospinal tract, enabling fine control and voluntary modification of gaits, similar to the rubrospinal tract [10]. Proximal musculature is mainly controlled by the reticulospinal tract and vestibulospinal tract [14] and works to maintain a standing posture. Different motor circuits may have different recovery processes after SCI, explaining the different motor performances in different limb positions. Under these circumstances, exploring motor performances of different limb segments is believed to be helpful in uncovering specific recovery processes of different descending motor pathways.

Principal component analysis (PCA) is a linear multivariate analysis that is used for data reduction and classification and has been widely used in studies of human locomotion $[8,25]$. For instance, PCA is conducted for the relevant matrices to produce new variables (i.e., principal components, PCs) that are used to substitute numerous originally correlated variables of movement. 
The first principal component (PC1) has the largest possible variance (i.e., accounts for the most of the variability in the data among all principal components), which would be the most efficient representation of the original database, and we sought it as the main locomotion kinematic parameter of each segment in this study.

We hypothesized that different hindlimb positions may display different locomotor recovery processes after SCI. The motor performance of monkey hindlimbs was analyzed after thoracic hemisection injury in terms of control hierarchy [9], which is defined as the variation degree of gait characteristics during movement; that is, the higher the control level is, the slighter is the kinematic characteristic variation [12]. To assess the recovery processes in different positions of different hierarchies, we also adopted PC1 as the representation of kinematic performance in each limb part and used the PC1 score as the evaluation standard. The results indicate that, after SCI, different hindlimb positions may display different locomotor recovery processes.

\section{Materials and Methods}

\section{Preparation}

Four adult female rhesus monkeys (Macaca mulatta) (age, $5.5 \pm 0.4$ years; weight, $5.9 \pm 0.7 \mathrm{~kg}$ ) were housed individually in single stainless steel cages $(72 \times 61 \times 87$ $\mathrm{cm})$ at $22-25^{\circ} \mathrm{C}$. All procedures that were performed involving animals were in accordance with the ethical standards of the Animal Ethics Committee of Capital Medical University. All monkeys received a unilateral hemisection at T7-T9 on the right side of the spinal cord, with $10 \mathrm{~mm}$ of spinal cord tissue being removed.

\section{Data collection}

The monkeys received treadmill training with a previously reported training and fixing method one month before surgery [31]. Treadmill locomotion was recorded for the first time one week after training, and this was repeated at 6 and 12 weeks post-lesion. Leg kinematics was recorded with a six-camera VICON motion system (VICON Motion System Ltd., Oxford, UK ; sampling at $100 \mathrm{~Hz}$ ). Seven reflective markers were secured to the shaved skin of the injured right leg in the following positions: the anterior superior iliac spine, posterior superior iliac spine, two-thirds of the femur, knee, ankle, heel, and second metatarsophalangeal joint (MTP joint). The treadmill speed was set at $0.139,0.222$, and $0.306 \mathrm{~m} / \mathrm{s}$.
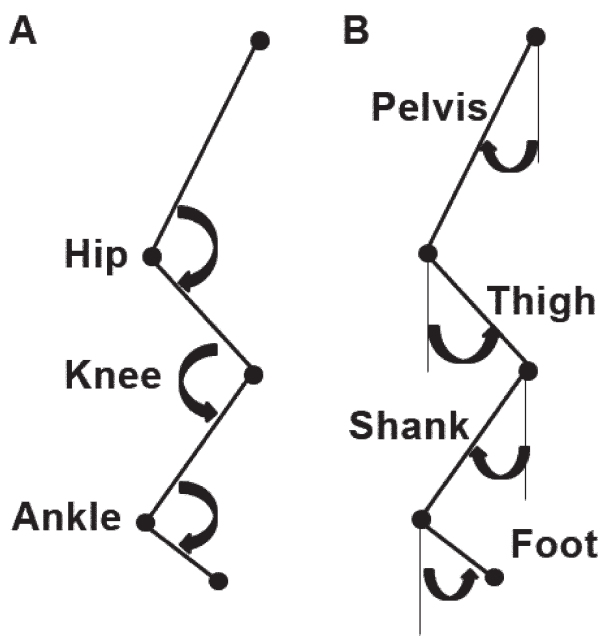

Fig. 1. Measurement of kinematics. A: Definitions of joint angles. Hip, knee, and ankle joint angles were defined as decreasing during flexion. B: Definitions of elevation angles. Elevation angles were defined as decreasing during clockwise rotation of the segments with respect to the vertical axis.

Each locomotor session consisted of 10 locomotor trials (one session at each speed). Data acquisition has been detailed previously [36].

\section{Data processing}

The joint angles of the hip, knee, and ankle were calculated based on the coordinate position of each marker (Fig. 1A). The elevation angle of each segment was defined as the angle between each segment and the vertical gravity axis [13], except for the elevation angle of the pelvis, which was defined as the angle between the vertical gravity axis and the line from the hip to the anterior superior iliac crest (Fig. 1B). Gait parameters are shown in Supplementary Table 1. The methods of calculating gait parameters are described in the supplementary materials.

\section{Principal components and statistics analysis}

PCA was adopted to analyze the locomotor performance of the limb endpoint and each joint, as well as the elevation angle of each segment. Each relevant matrix was composed of all module parameters at each time point (Supplementary Table 1). PC1 scores were averaged at each time point and illustrated in a bar graph. Factor loadings with greater correlation were extracted (the absolute value of factor loading was larger than 0.75 [2]); from these, the most representative parameters were 
sequentially selected and illustrated in a bar graph.

One-way analysis of variance (ANOVA) with Tukey's HSD and Dunnett's T3 post hoc comparison were used to test the PC1 scores of each joint, elevation angle of each segment, and limb endpoint trajectory at each time point, as well as the differences in representative parameters. Pearson correlation analysis was conducted for the PC scores of the elevation angle of each segment and limb endpoint trajectory. Statistical analysis was conducted with SPSS version 19.0 (IBM Corp., Armonk, NY, USA), and all quantitative data were expressed as means \pm SE. $P<0.05$ was considered statistically significant.

\section{Results}

In the present study, leg kinematics in treadmill locomotion were recorded for four monkeys before surgery, as well as 6 and 12 weeks after surgery. Valid data were obtained at $0.139,0.222$, and $0.306 \mathrm{~m} / \mathrm{s}$ for three monkeys, while valid data were only obtained at $0.222 \mathrm{~m} / \mathrm{s}$ for the other one. A total of 300 intact gait cycles of the right hindlimb ( 3 monkeys $\times 3$ speeds $\times 10$ gait cycles $\times 3$ time points and 1 monkey $\times 1$ speed $\times 10$ gait cycles $\times 3$ time points) were chosen for data analysis.

\section{Limb endpoint}

Motor performance was recorded and reconstructed before surgery and 6 and 12 weeks post-lesion (Fig. 2A). At 6 weeks post-lesion, the right hindlimb drag was remarkable and was accompanied by smaller limb endpoint displacement without valid forward stepping. At 12 weeks post-lesion, the right hindlimb was observed to have obvious forward stepping but was still accompanied by partial drag. Massive limb flexion was present, indicating that there might be a compensating trunk mechanism working to initiate movement. PC analysis was conducted to compare the motor performance of the limb endpoint at each time point (Fig. 2B). A significant difference was found at all three time points (all time points: $P<0.001)$. Compared to 6 weeks post-lesion, PC1 was closer to the pre-lesion value at 12 weeks post-lesion (Fig. 2B), implying partial recovery of the limb endpoint path. Furthermore, the correlation between each variable and $\mathrm{PC} 1$ was extracted from factor loading. Representative parameters were extracted from highly correlated parameters and illustrated in a bar graph. Step length, height, and limb endpoint trajectory were partially re- covered at 12 weeks post-lesion (all time points: step length, all: $P<0.001$; step height, $P<0.001$; path length, $P<0.001$; Fig. 2C-E).

\section{Segments}

The elevation angle of each segment was analyzed with the same method. We first analyzed the pelvis segment. The pelvis segment at 12 weeks post-lesion was significantly different from that at 6 weeks post-lesion as well as from that of the healthy stage, without any motor recovery (intact vs. 6 weeks, $P<0.001$; intact vs. 12 weeks, $P<0.05$; 6 weeks vs. 12 weeks, $P<0.001$; Fig. $3 \mathrm{~A})$. The forward, backward, and average of the pelvis elevation angles all showed a significant difference at the three time points. At 6 weeks post-lesion, the hip swung slightly, keeping the body in an upright posture. At 12 weeks post-lesion, the hip showed an obvious forward pelvis compared with the status before surgery (pelvis backward, $P<0.001, P<0.01$, and $P<0.001$ for intact vs. 6 weeks, intact vs. 12 weeks, and 6 weeks vs. 12 weeks, respectively; Pelvis forward, $P<0.001$ for intact vs. 6 weeks and 6 weeks vs. 12 weeks; Pelvis average, $P<0.001, P<0.05, P<0.001$ for intact vs. 6 weeks, intact vs. 12 weeks, and 6 weeks vs. 12 weeks, respectively; Fig. 2A).

The PC1 values of the thigh segment at 6 and 12 weeks post-lesion were not significantly different but were both significantly different from the intact level $(P<0.001$, for intact vs. 6 weeks and intact vs. 12 weeks; Fig. 3B). After SCI, the amplitude of thigh forward and backward oscillation generally decreased, and the thigh tended toward a vertical position (thigh backward, $P<0.05$ for intact vs. 12 weeks; Thigh forward, $P<0.001$ for intact vs. 6 weeks and intact vs. 12 weeks; Thigh average, $P<0.001$ for intact vs. 6 weeks and intact vs. 12 weeks; Fig. 3B). This suggests that no remarkable kinematics recovery occurred at the thigh segment.

The PC1 values of the elevation angle of the shank segment showed partial kinematic recovery at 12 weeks post-lesion (all time points: $P<0.001$; Fig. $3 \mathrm{C}$ ). At 6 weeks post-lesion, the amplitude of shank backward and forward oscillation was decreased, most likely because of stronger drag. At 12 weeks post-lesion, the elevation angle of the shank was increased, indicating reduced drag (Shank backward, $P<0.001$ for all time points; Shank forward, $P<0.001$ for all time points; Shank average, $P<0.001$ for all time points; Fig. $3 \mathrm{C}$ ).

The elevation angle of the foot segment showed a 
A
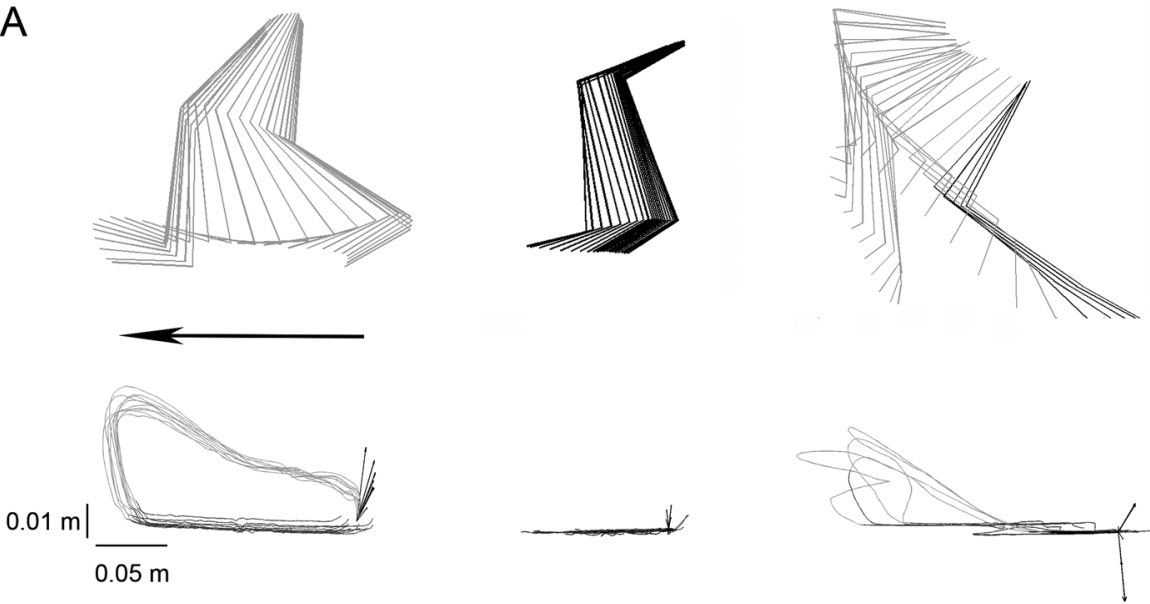

B Limb endpoint PC1 (variance: $35 \%$ ) (a.u.)

Step length (m)
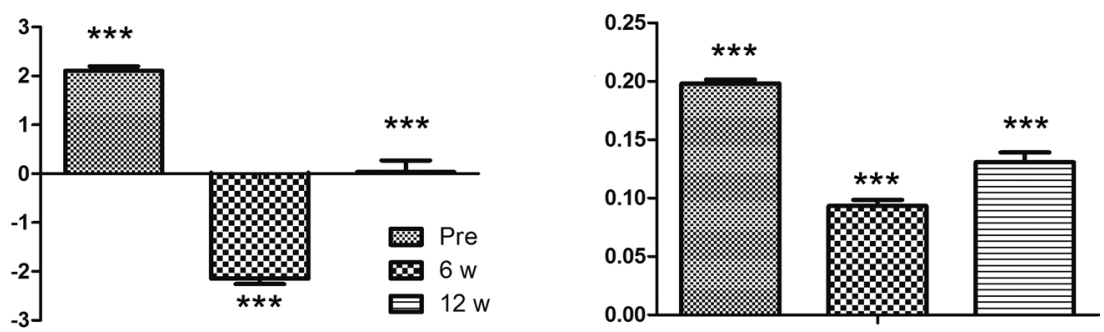

Step height (m)

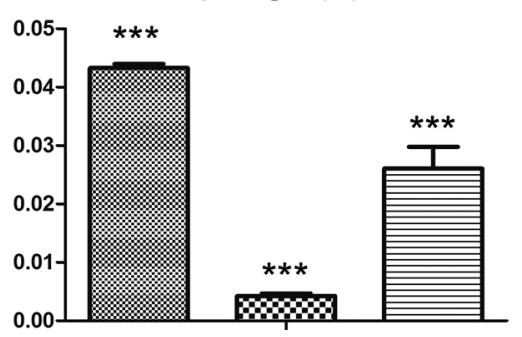

Path length (m)

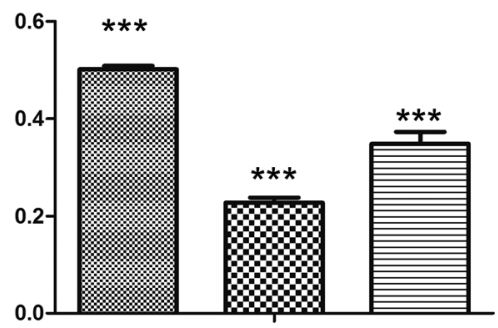

Fig. 2. Recovery of the limb endpoint. A: Representative stick decompositions of lesion-side hindlimb movements at the swing phase during stepping on the treadmill at $0.222 \mathrm{~m} / \mathrm{s}$ before and 6 and 12 weeks after the lesion. The trajectories ( $n=10$ steps; grey, normal gait; black, drag) of the limb endpoint are shown together with the intensity and direction of the limb endpoint velocities (arrows) at swing onset. B: Bar graph of average scores on PC1 of the limb endpoint. C: Mean values of the parameters with high factor loadings on PC1 of the limb endpoint. $* P<0.05$ (ANOVA), ${ }^{* *} P<0.01$ (ANOVA), $* * * P<0.001$ (ANOVA). Data are means \pm SEM. Pre, before surgery; w, weeks.

similar variation trend to that of the shank (all time points: $P<0.001$; Fig. 3D). At 12 weeks post-lesion, foot backward and forward were both positive, indicating reduced drag and partially increased back flexion ability (foot backward, $P<0.001$ for intact vs. 6 weeks and 6 weeks vs. 12 weeks; Foot forward, $P<0.001$ for all time points; Foot average, $P<0.001, P<0.01$, and $P<0.001$ for intact vs. 6 weeks, intact vs. 12 weeks and 6 weeks vs. 12 weeks, respectively; Fig. 3D).

The motor performances of all three hindlimb segments at 12 weeks post-lesion were compared with those in the intact status. The closer the motor performance was to 1 , the closer the performance was to intact status. Our results showed that the value gradually approached 

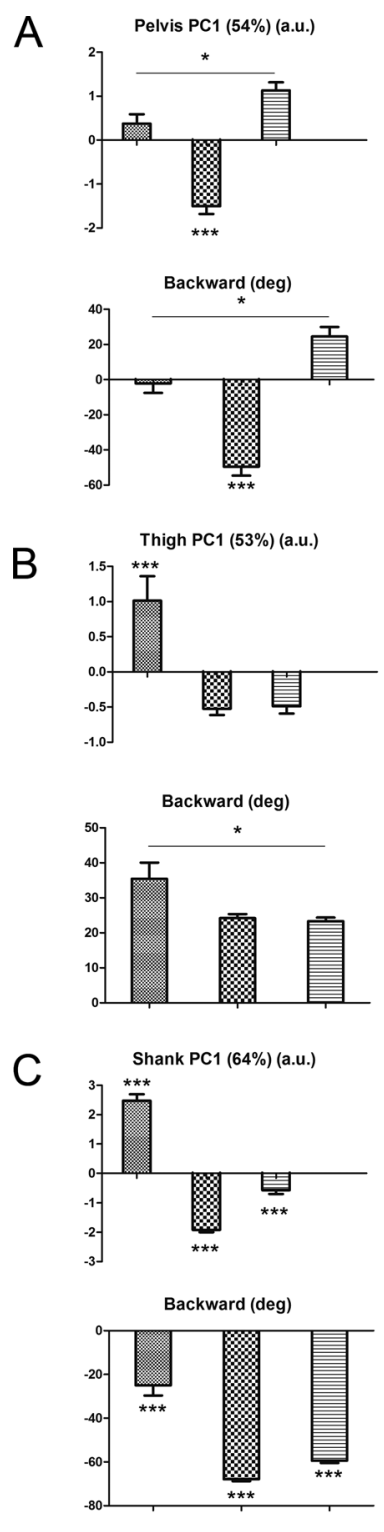

D

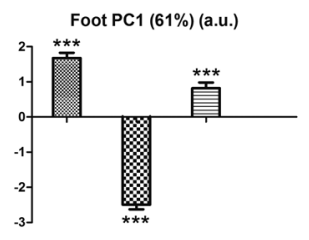

Backward (deg)

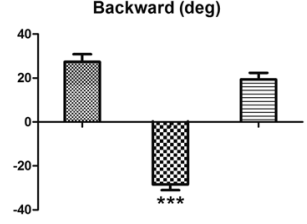

$E$

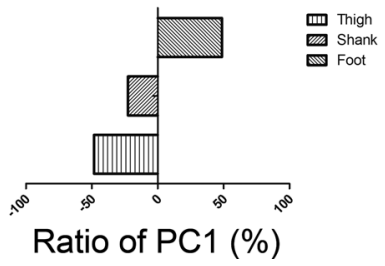

Forward (deg)
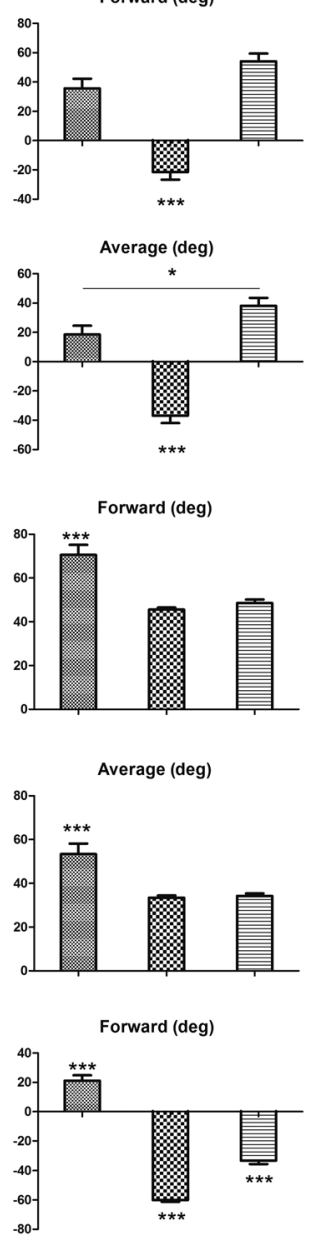

Average (deg)

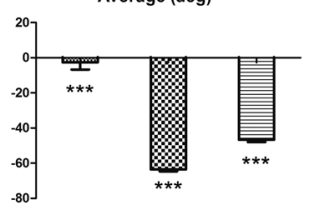

Forward (deg)
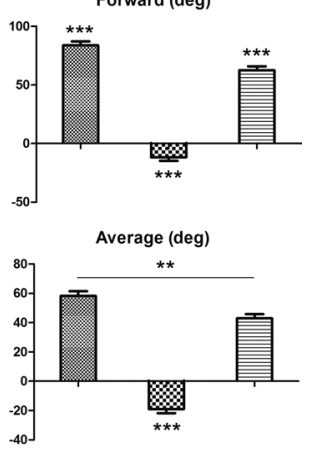

1 from the proximal limb to the distal limb and that this was accompanied by gradually more extensive motor performance recovery (Fig. 3E).

Joints

$\mathrm{PC}$ analysis of the hip joint showed that $\mathrm{PC} 1$ at 12 weeks post-lesion was significantly different from the intact value and the value at 6 weeks post-lesion. The difference in motor performance became remarkable over time, and no kinematic recovery occurred $(P<0.001$ for intact vs. 12 weeks and 6 weeks vs. 12 weeks; Fig. $4 \mathrm{~A})$.

The kinematic performance of the knee joint was recovered to some degree at 12 weeks post-lesion (all time points: $P<0.001$ ) (Fig. 4B). At that time, knee flexion ability was strengthened, and in general, the knee performance in the stance phase showed no difference from the intact status (knee extension, $P<0.001$ for 6 weeks vs. 12 weeks; knee average, $P<0.001$ for 6 weeks vs. 12 weeks; knee stance, $P<0.001$ for intact vs. 6 weeks and 6 weeks vs. 12 weeks; Fig. 4B).

For the motor performance of the ankle joint, the PC1 value at 12 weeks post-lesion was not significantly different from that in the intact status, showing relatively good kinematic recovery $(P<0.001$ for intact vs. 6 weeks; $P<0.01$ for 6 weeks vs. 12 weeks; Fig. 3C). At 12 weeks post-lesion, ankle flexion ability was partially recovered, and the joint average and ankle swing average showed no significant difference from the intact values (Ankle flexion, $P<0.001$ for all time points; Ankle average, $P<0.001$ for intact vs. 6 weeks and 6 weeks vs. 12 weeks; Ankle swing, $P<0.001$ for intact vs. 6 weeks and 6 weeks vs. 12 weeks; Fig. 4C).

The comparison of the motor performances of these three joints at 12 weeks post-lesion indicated that the performance of the ankle joint was closer to the normal status, while the hip joint performed the worst (Fig. 4D). This implies that motor defects gradually decreased from the proximal limb to the distal limb.

Fig. 3. Recovery of hindlimb segments. A: Bar graph of average scores on $\mathrm{PC} 1$ of the pelvis (A), thigh (B), shank (C), and foot (D). Mean values of the representative parameters with high factor loadings on $\mathrm{PC} 1$ of the pelvis (A), thigh (B), shank (C), and foot (D). E: Comparison of recovery status of each segment at 12 weeks post-lesion. Each value was the ratio of $\mathrm{PC} 1$ at 12 weeks post-lesion to presurgery PC1. $* P<0.05$ (ANOVA), $* * P<0.01$ (ANOVA), $* * * P<0.001$ (ANOVA). Data are means \pm SEM. Deg, degrees; a.u., arbitrary units. 
Finally, correlation analysis was conducted between the PC1 scores of the limb endpoint and each hindlimb segment. The results showed that the limb endpoint was correlated with all segments, especially with the foot, followed by the shank and the thigh (thigh, $\mathrm{r}=0.462$, $P<0.001$; shank, $\mathrm{r}=0.818, P<0.001$; foot, $\mathrm{r}=0.824$, $P<0.001$; Fig. 5A-C).

\section{Discussion}

In the present study, kinematics data were assessed before surgery, as well as 6 and 12 weeks post-lesion. After a period of time, the motor function was spontaneously recovered to some degree. Interestingly, the distal limb might have more extensive functional recovery than the proximal limb. The discovery of different locomotor recovery processes from the proximal limb to the distal limb may help further understand the spinal cord plasticity after SCI.

Monkeys that received a thoracic hemisection lesion exhibited sustained locomotor deficits. All animals showed severe hemiplegic paralysis 1 day after injury and lay on the floor in a prone position. The most obvious deficit was paw drag, which was reflected in reduced ankle dorsiflexion during the swing phase of gait. As the animals recovered, they showed slight to extensive movements of the lesion leg. At 12 week post-lesion, the monkeys showed recovery in step height, step length, and the elevation and joint angles of the hindlimb. Functional recovery comprised reorganization in the spinal cord and at the superspinal level.

Previous research evaluated motor function recovery after SCI in various aspects, including general hindlimb motor performance [26], intra-limb coordination $[1,2]$, inter-limb coordination [7], and control hierarchy [12]. While several research studies have focused on general hindlimb performance, studies based on different control hierarchies have enabled the sensitive reflection of different performances of different hindlimb parts. Here, we adopted the control hierarchy theory to analyze motor performance in terms of limb endpoint movement, hindlimb segments, and joint angles. We found that locomotor recovery performance in all three hierarchies at 12 weeks post-lesion was partially recovered compared with those at 6 weeks post-lesion. Moreover, we observed that in the latter two hierarchies, motor performance at the distal limb was closer to the normal level than at the proximal limb, showing extensive locomotor recovery. Courtine et al. [7] reported that for monkey models of a unilateral thoracic corticospinal tract lesion, the shank muscle EMG amplitude returned to the prelesion baseline at 12 weeks post-lesion, while the vastus lateralis did not show great recovery. The distal muscle showed better recovery than did the proximal.

Distal musculature is mainly controlled by the corticospinal tract, which enables fine control and voluntary modification of gaits, similar to the rubrospinal tract [10]. Proximal musculature is mainly controlled by the reticulospinal tract and the vestibulospinal tract [14] and works to maintain the standing posture. We speculate that more extensive locomotor recovery at the distal limb may be attributed to plasticity reorganization of the motor circuits that are mainly composed of the corticospinal tract. After incomplete SCI, plasticity may vary in the cortex and spinal cord of the motor system, where the corticospinal tract resides [22]. All reorganized plasticity benefits the reorganization of the motor circuits that control the distal limb.

Of note, Donati et al. [9] reported that after brainmachine interface-based gait protocol training, SCI patients in the chronic phase could have recovery that progressed from proximal to distal muscles, in contrast with our results. As is well known, spontaneous recovery occurs mainly during the first 3 months after SCI. Therefore, we hypothesize that their recovery resulted from rehabilitation training, while our results were due to spontaneous recovery.

For limb endpoint recovery, limb endpoint kinematics indicates that the motor performance of the highest hierarchy directly reflects kinematic goals [21]. It has been reported that after SCI, the limb endpoint was the first to show motor performance similar to the normal status [12]. As revealed by the correlation analysis between the PC1 scores of the limb endpoint and elevation angles of each segment, limb endpoint variation was correlated with all segments, especially the foot segment. Although limb endpoint kinematics is considered to be a comprehensive reflection of all hindlimb segments, it may be most directly impacted by the foot segment, because the positions of the foot segment and limb endpoint are relatively close in terms of biological structure. Because the kinematic variation in the foot segment was most closely correlated with that of the limb endpoint, the motor performance of the distal end had a greater effect on the recovery of the limb endpoint, thus explaining why the limb endpoint had a more extensive functional 

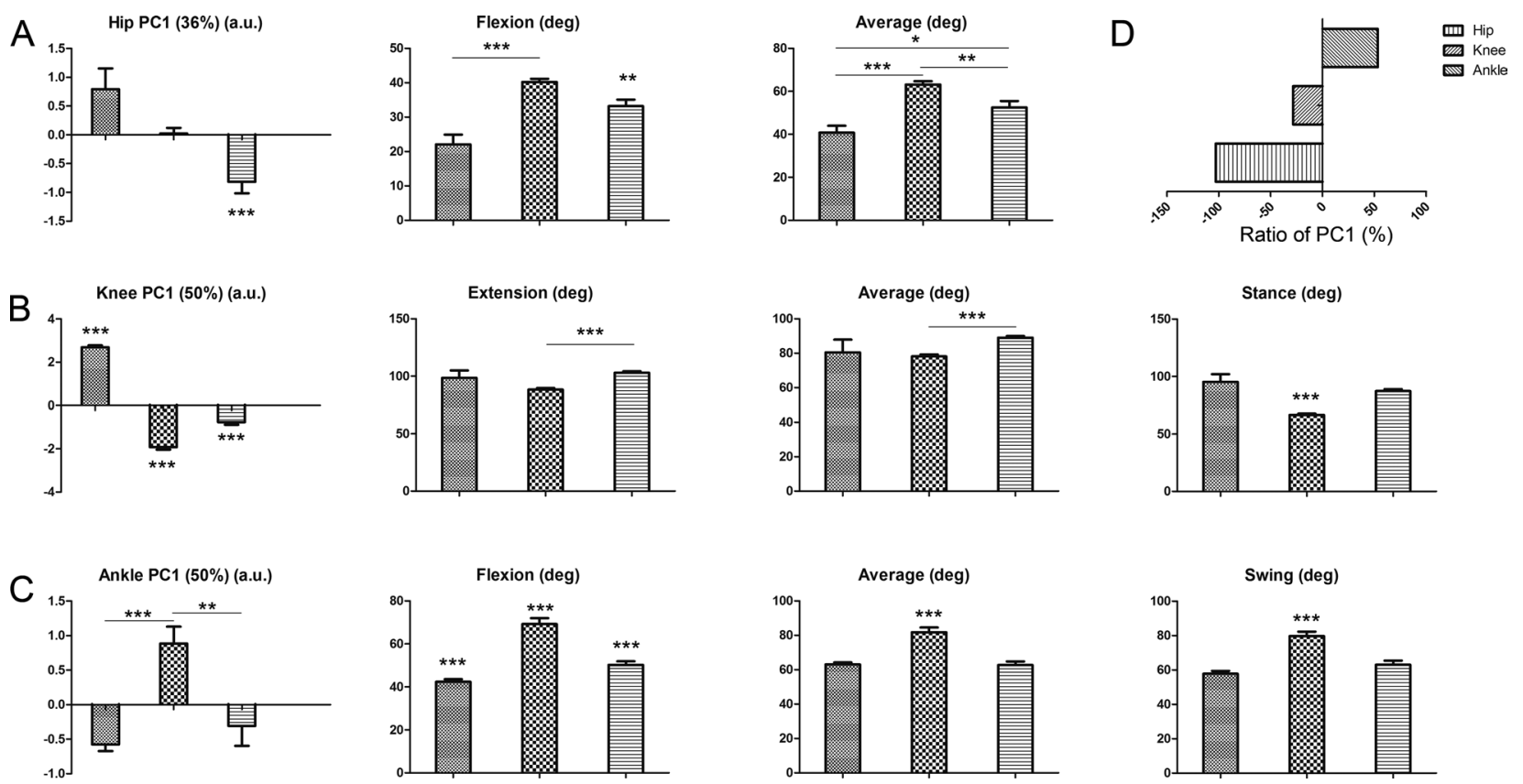

Fig. 4. Recovery of hindlimb joints. A: Bar graph of average scores on $\mathrm{PC} 1$ of the hip (A), knee (B), and ankle joint (C). Mean values of the representative parameters with high factor loadings on PC1 of the hip (A), knee (B), and ankle (C). D: Comparison of the recovery status of each segment at 12 weeks post-lesion. ${ }^{*} P<0.05$ (ANOVA), ${ }^{*} P<0.01$ (ANOVA), $* * * P<0.001$ (ANOVA). Data are means \pm SEM. Deg, degrees; a.u., arbitrary units.
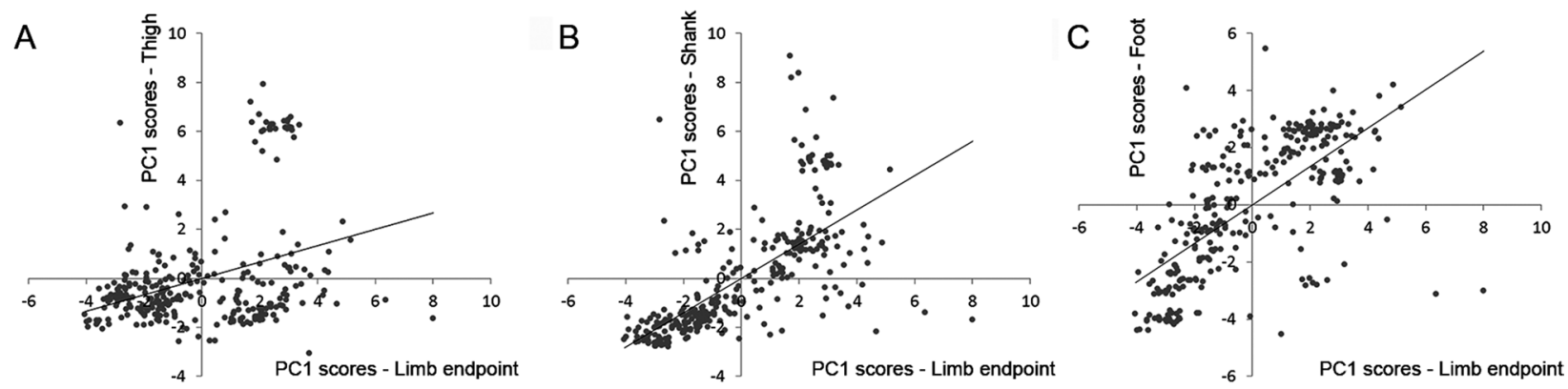

Fig. 5. Linear regression plots that demonstrate the correlation between $\mathrm{PC} 1$ scores of the limb endpoint and thigh (A), shank (B), and foot (C) at 12 weeks post-lesion. Each circle represents a gait cycle (300 cycles in total).

recovery.

We also found that, unlike the segments with remarkable locomotor recovery, the pelvis segment showed a constant increase in forward degree, indicating that the SCI monkeys probably used a strategy distinct from the normal one. Grasso et al. [12] reported that SCI patients changed their foot kinematics by strengthening the movement of their trunk and pelvis as a new motor strategy after rehabilitation training. We deduce that the monkeys in this study probably used a similar strategy to compensate for locomotor deficits and complete forward stepping in a new way.
There are some limitations in this study. First, the sample number was too small to avoid the influence of individual differences on the results. Second, musculature variation was not detected in terms of EMG activity in a higher hierarchy, so we could not illustrate the recovery status of each hindlimb part more clearly. In future research, we will further explore the locomotor recovery processes of different hindlimb parts by analyzing and discussing the EMG activity of musculature.

In the present study, we analyzed the functional locomotor performance of rhesus monkeys and observed partial locomotor recovery after SCI that might be more 
extensive at the distal limb. The results revealed that different hindlimb parts had different locomotor recovery processes. This may indicate the plasticity of motor circuits after SCI, as well as rehabilitation training for SCI patients in clinical trials.

\section{Conflict of Interest}

Financial disclosure statements have been obtained, and no conflicts of interest have been reported by the authors or by any individuals in control of the content of this article.

\section{Acknowledgment}

This work was supported by National Natural Science Foundation of China (Grants 31730030, 31650001, 31670988, 31320103903), Ministry of Science and Technology of China (Grants 2017YFC1104001, 2017YFC1104002), Beijing Science and Technology Program (Grants Z181100001818007), Beijing Natural Science Foundation Program (Grant KZ201810025030).

\section{References}

1. Awai, L., Bolliger, M., Ferguson, A.R., Courtine, G., and Curt, A. 2016. Influence of spinal cord integrity on gait control in human spinal cord injury. Neurorehabil. Neural Repair 30: 562-572. [Medline] [CrossRef]

2. Awai, L. and Curt, A. 2016. Locomotor recovery in spinal cord injury - insights beyond walking speed and distance. $J$. Neurotrauma 33: 1428-1435. [Medline] [CrossRef]

3. Babu, R.S. and Namasivayam, A. 2008. Recovery of bipedal locomotion in bonnet macaques after spinal cord injury: footprint analysis. Synapse 62: 432-447. [Medline] [CrossRef]

4. Bachmann, L.C., Matis, A., Lindau, N.T., Felder, P., Gullo, M., and Schwab, M.E. 2013. Deep brain stimulation of the midbrain locomotor region improves paretic hindlimb function after spinal cord injury in rats. Sci. Transl. Med. 5: 208ra146. [Medline] [CrossRef]

5. Ballermann, M. and Fouad, K. 2006. Spontaneous locomotor recovery in spinal cord injured rats is accompanied by anatomical plasticity of reticulospinal fibers. Eur. J. Neurosci. 23: 1988-1996. [Medline] [CrossRef]

6. Courtine, G., Bunge, M.B., Fawcett, J.W., Grossman, R.G., Kaas, J.H., Lemon, R., Maier, I., Martin, J., Nudo, R.J., Ramon-Cueto, A., Rouiller, E.M., Schnell, L., Wannier, T., Schwab, M.E., and Edgerton, V.R. 2007. Can experiments in nonhuman primates expedite the translation of treatments for spinal cord injury in humans? Nat. Med. 13: 561-566. [Medline] [CrossRef]

7. Courtine, G., Roy, R.R., Raven, J., Hodgson, J., McKay, H.,
Yang, H., Zhong, H., Tuszynski, M.H., and Edgerton, V.R 2005. Performance of locomotion and foot grasping following a unilateral thoracic corticospinal tract lesion in monkeys (Macaca mulatta). Brain 128: 2338-2358. [Medline] [CrossRef]

8. Deluzio, K.J., Wyss, U.P., Zee, B., Costigan, P.A., and Serbie, C. 1997. Principal component models of knee kinematics and kinetics: normal vs. pathological gait patterns. Hum. Mov. Sci. 16: 201-217. [CrossRef]

9. Donati, A.R.C., Shokur, S., Morya, E., Campos, D.S., Moioli, R.C., Gitti, C.M., Augusto, P.B., Tripodi, S., Pires, C.G., Pereira, G.A., Brasil, F.L., Gallo, S., Lin, A.A., Takigami, A.K., Aratanha, M.A., Joshi, S., Bleuler, H., Cheng, G., Rudolph, A., and Nicolelis, M.A. 2016. Long-Term Training with a Brain-Machine Interface-Based Gait Protocol Induces Partial Neurological Recovery in Paraplegic Patients. Sci. Rep. 6: 30383. [Medline] [CrossRef]

10. Drew, T., Jiang, W., and Widajewicz, W. 2002. Contributions of the motor cortex to the control of the hindlimbs during locomotion in the cat. Brain Res. Brain Res. Rev. 40: 178-191. [Medline] [CrossRef]

11. Friedli, L., Rosenzweig, E.S., Barraud, Q., Schubert, M., Dominici, N., Awai, L., Nielson, J.L., Musienko, P., NoutLomas, Y., Zhong, H., Zdunowski, S., Roy, R.R., Strand, S.C., van den Brand, R., Havton, L.A., Beattie, M.S., Bresnahan, J.C., Bézard, E., Bloch, J., Edgerton, V.R., Ferguson, A.R., Curt, A., Tuszynski, M.H., and Courtine, G. 2015. Pronounced species divergence in corticospinal tract reorganization and functional recovery after lateralized spinal cord injury favors primates. Sci. Transl. Med. 7: 302ra134. [Medline] [CrossRef]

12. Grasso, R., Ivanenko, Y.P., Zago, M., Molinari, M., Scivoletto, G., Castellano, V., Macellari, V., and Lacquaniti, F. 2004. Distributed plasticity of locomotor pattern generators in spinal cord injured patients. Brain 127: 1019-1034. [Medline] [CrossRef]

13. Grasso, R., Zago, M., and Lacquaniti, F. 2000. Interactions between posture and locomotion: motor patterns in humans walking with bent posture versus erect posture. J. Neurophysiol. 83: 288-300. [Medline] [CrossRef]

14. Kuypers, H.G.J.M. 1981. Anatomy of the descending pathways. In: Brooks VBV, Brookhart JM, Mountcastle VB, editors. Handbook of physiology, section 1: the nervous system. Vol 2. Bethesda: American Physiological Society. pp:597666.

15. Lawrence, D.G. and Kuypers, H.G. 1968. The functional organization of the motor system in the monkey. II. The effects of lesions of the descending brain-stem pathways. Brain 91: 15-36. [Medline] [CrossRef]

16. Little, J.W., Ditunno, J.F. Jr., Stiens, S.A., and Harris, R.M. 1999. Incomplete spinal cord injury: neuronal mechanisms of motor recovery and hyperreflexia. Arch. Phys. Med. Rehabil. 80: 587-599. [Medline] [CrossRef]

17. Maier, I.C. and Schwab, M.E. 2006. Sprouting, regeneration and circuit formation in the injured spinal cord: factors and activity. Philos. Trans. R. Soc. Lond. B Biol. Sci. 361: 1611-1634. [Medline] [CrossRef]

18. Martinez, M., Delivet-Mongrain, H., Leblond, H., and Ros- 
signol, S. 2011. Recovery of hindlimb locomotion after incomplete spinal cord injury in the cat involves spontaneous compensatory changes within the spinal locomotor circuitry. J. Neurophysiol. 106: 1969-1984. [Medline] [CrossRef]

19. Martinez, M., Delivet-Mongrain, H., and Rossignol, S. 2013. Treadmill training promotes spinal changes leading to locomotor recovery after partial spinal cord injury in cats. J. Neurophysiol. 109: 2909-2922. [Medline] [CrossRef]

20. Musienko, P.E., Bogacheva, I.N., and Gerasimenko, Y.P. 2007. Significance of peripheral feedback in the generation of stepping movements during epidural stimulation of the spinal cord. Neurosci. Behav. Physiol. 37: 181-190. [Medline] [CrossRef]

21. Poppele, R. and Bosco, G. 2003. Sophisticated spinal contributions to motor control. Trends Neurosci. 26: 269-276. [Medline] [CrossRef]

22. Raineteau, O. and Schwab, M.E. 2001. Plasticity of motor systems after incomplete spinal cord injury. Nat. Rev. Neurosci. 2: 263-273. [Medline] [CrossRef]

23. Rao, J.S., Liu, Z., Zhao, C., Wei, R.H., Zhao, W., Yang, Z.Y., and Li, X.G. 2016. Longitudinal evaluation of functional connectivity variation in the monkey sensorimotor network induced by spinal cord injury. Acta Physiol. (Oxf.) 217: 164173. [Medline] [CrossRef]

24. Rao, J.S., Manxiu, M., Zhao, C., Xi, Y., Yang, Z.Y., Zuxiang, L., and Li, X.G. 2013. Atrophy and primary somatosensory cortical reorganization after unilateral thoracic spinal cord injury: a longitudinal functional magnetic resonance imaging study. BioMed Res. Int. 2013: 753061. [Medline] [CrossRef]

25. Rein, R., Button, C., Davids, K., and Summers, J. 2010. Cluster analysis of movement patterns in multiarticular actions: a tutorial. Mot. Contr. 14: 211-239. [Medline] [CrossRef]

26. Rosenzweig, E.S., Courtine, G., Jindrich, D.L., Brock, J.H., Ferguson, A.R., Strand, S.C., Nout, Y.S., Roy, R.R., Miller, D.M., Beattie, M.S., Havton, L.A., Bresnahan, J.C., Edgerton, V.R., and Tuszynski, M.H. 2010. Extensive spontaneous plasticity of corticospinal projections after primate spinal cord injury. Nat. Neurosci. 13: 1505-1510. [Medline] [CrossRef]

27. Rossignol, S., Chau, C., Brustein, E., Bélanger, M., Barbeau, H., and Drew, T. 1996. Locomotor capacities after complete and partial lesions of the spinal cord. Acta Neurobiol. Exp. (Warsz.) 56: 449-463. [Medline]

28. Scheibel, M.E. and Scheibel, A.B. 1966. Terminal axonal patterns in cat spinal cord. I. The lateral corticospinal tract. Brain Res. 2: 333-350. [Medline] [CrossRef]

29. Suresh Babu, R., Sunandhini, R.L., Sridevi, D., Periasamy, P., and Namasivayam, A. 2012. Locomotor behavior of bonnet monkeys after spinal contusion injury: footprint study. Synapse 66: 509-521. [Medline] [CrossRef]

30. Takeoka, A., Vollenweider, I., Courtine, G., and Arber, S. 2014. Muscle spindle feedback directs locomotor recovery and circuit reorganization after spinal cord injury. Cell 159: 1626-1639. [Medline] [CrossRef]

31. Wei, R.H., Song, W., Zhao, C., Zhao, W., Li, L.F., Ji, R., Rao, J.S., Yang, Z.Y., and Li, X.G. 2016. Influence of walking speed on gait parameters of bipedal locomotion in rhesus monkeys. J. Med. Primatol. 45: 304-311. [Medline] [CrossRef]

32. Weidner, N., Ner, A., Salimi, N., and Tuszynski, M.H. 2001. Spontaneous corticospinal axonal plasticity and functional recovery after adult central nervous system injury. Proc. Natl. Acad. Sci. USA 98: 3513-3518. [Medline] [CrossRef]

33. Zhao, C., Rao, J.S., Pei, X.J., Lei, J.F., Wang, Z.J., Yang, Z.Y., and Li, X.G. 2016. Longitudinal study on diffusion tensor imaging and diffusion tensor tractography following spinal cord contusion injury in rats. Neuroradiology 58: 607-614. [Medline] [CrossRef]

34. Zhao, C., Song, W., Rao, J.S., Zhao, W., Wei, R.H., Zhou, X., Tian, P.Y., Yang, Z.Y., and Li, X.G. 2017. Combination of kinematic analyses and diffusion tensor tractrography to evaluate the residual motor functions in spinal cord-hemisected monkeys. J. Med. Primatol. 46: 239-247. [Medline] [CrossRef]

35. Zhao, C., Rao, J.S., Pei, X.J., Lei, J.F., Wang, Z.J., Zhao, W., Wei, R.H., Yang, Z.Y., and Li, X.G. 2018. Diffusion tensor imaging of spinal cord parenchyma lesion in rat with chronic spinal cord injury. Magn. Reson. Imaging 47: 25-32. [Medline] [CrossRef]

36. Zhao, W., Song, W., Rao, J.S., Wei, R.H., Li L.F., Ji, R., Zhao C., Yang, Z.Y., and Li, X.G. 2018. Gait division of healthy and spinal cord-injury Rhesus monkeys by one-dimensional toe signals. J. Mech. Med. Biol. 18: 1850017. [CrossRef] 